\title{
ArchbotLit—-the archaeobotanical literature database: an update of the search engine for literature on archaeological remains of cultivated plants since 1981
}

\author{
Wiebke Kirleis $^{1}\left[\right.$ [ Helmut Kroll ${ }^{1} \cdot$ Tanja Reiser $^{1} \cdot$ Clemens Schmid $^{2} \cdot$ Kay Schmütz $^{1}$
}

Received: 2 March 2020 / Accepted: 17 August 2020 / Published online: 13 September 2020

(c) The Author(s) 2020

\begin{abstract}
The online Archaeobotanical Literature Database (ArchbotLit) is an important tool for getting targeted access to archaeobotanical publications. It offers the opportunity for archaeobotanists, archaeologists, freelancers, students and an interested public to easily obtain information about cultivated plants and their development. In addition it increases the visibility of archaeobotanical studies beyond the inner circle and supports teaching in environmental archaeology. The ArchbotLit database builds upon the efforts of Jürgen Schultze-Motel, Gatersleben, and Helmut Kroll, Kiel, who have collected offprints of papers from archaeobotanists worldwide since the 1980s. The content was transferred into referenced plant lists that were published annually from 1992 to 2001 in the central organ of the "International Workgroup for Palaeoethnobotany (IWGP)", the journal "Vegetation History and Archaeobotany" and as contributions entitled "Literature on archaeological remains of cultivated plants". These were succeeded by the online database "archaeobotany.de", comprising the literature from 1981 to 2004. To sustain this online-database in the public domain it was recently converted into the wiki-platform "ArchbotLit", hosted at Kiel University. Here, we introduce the new wiki-online-database and its functionalities for data upload and as a search engine.
\end{abstract}

Keywords Literature database on archaeobotanical remains · Wiki-platform · Crops · Carpology $\cdot$ Archaeobotany

\section{Introduction}

Our knowledge about the (pre-) history of crop plants is largely based on fossil and subfossil plant remains from archaeological excavations. Literature on past crop plants is scattered in a variety of international journals, excavations reports, but also in so-called grey literature and is thus often difficult to access. Here we present the new wiki-platform Archaeobotanical Literature Database (ArchbotLit) hosted at Kiel University that was released prior to the IWGP conference in Lecce at the end of May 2019 (Fig. 1). The literature-based crop plant database ArchbotLit (Kirleis and

Communicated by F. Bittmann.

Wiebke Kirleis

wiebke.kirleis@ufg.uni-kiel.de

1 Institute for Pre- and Protohistoric Archaeology, CAU Kiel, Johanna-Mestorf-Straße 2-6, 24118 Kiel, Germany

2 MPI for the Science of Human History, Kahlaische Straße 10, 07745 Jena, Germany
Schmütz 2018) offers a central and sustainable platform for archaeobotanists, archaeologists, freelancers, students and the interested public world-wide to search for literature on crop species and their occurrence in specific archaeological periods or regions, including the option for multiple category search functions. For the informed archaeobotany community it further provides the opportunity to increase the visibility and accessibility of their own publications.

The bibliography of crop plants had already been initiated by Jürgen Schultze-Motel (Gatersleben, Germany) in the 1980s and published in the journal "Die Kulturpflanze" (e.g. Schultze-Motel 1983) and was continued from 1992 in "Vegetation History and Archaeobotany" (e.g. SchultzeMotel 1992). In 1995 (until 2001) Helmut Kroll took over (e.g. Kroll 1995). Then in the late 1990s, an online version of the database was established by Helmut Kroll, Rainer Pasternak (both Kiel, Germany) and Alexander Medović (Novi Sad, Serbia). This database comprised 9,422 single species data sets from literature on archaeological remains of cultivated plants from the years 1981 to 2004. ArchbotLit is now the follow-up of this online database. All former entries 
Fig. 1 The online Archaeobotanical Literature Database "ArchbotLit" at https://www. wikis.uni-kiel.de/archbotlit/

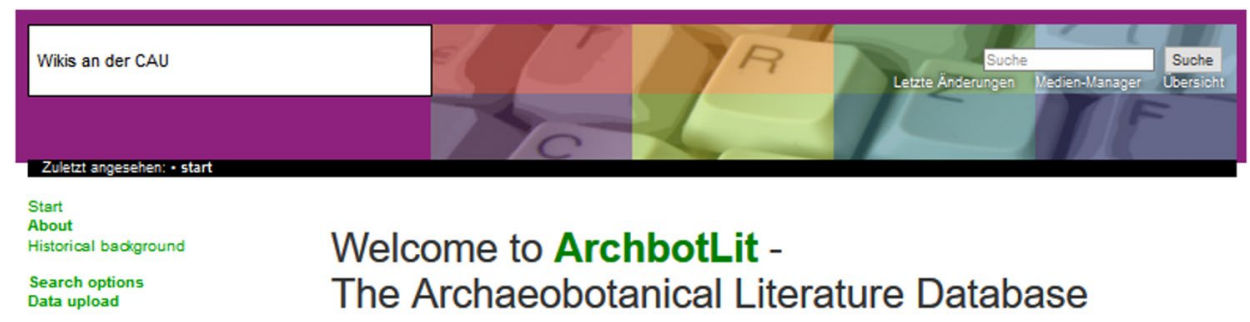

Taxalist

Synonyms

Downloads

Contact

Database for literature on archaeological remains of cultivated plants since 1981
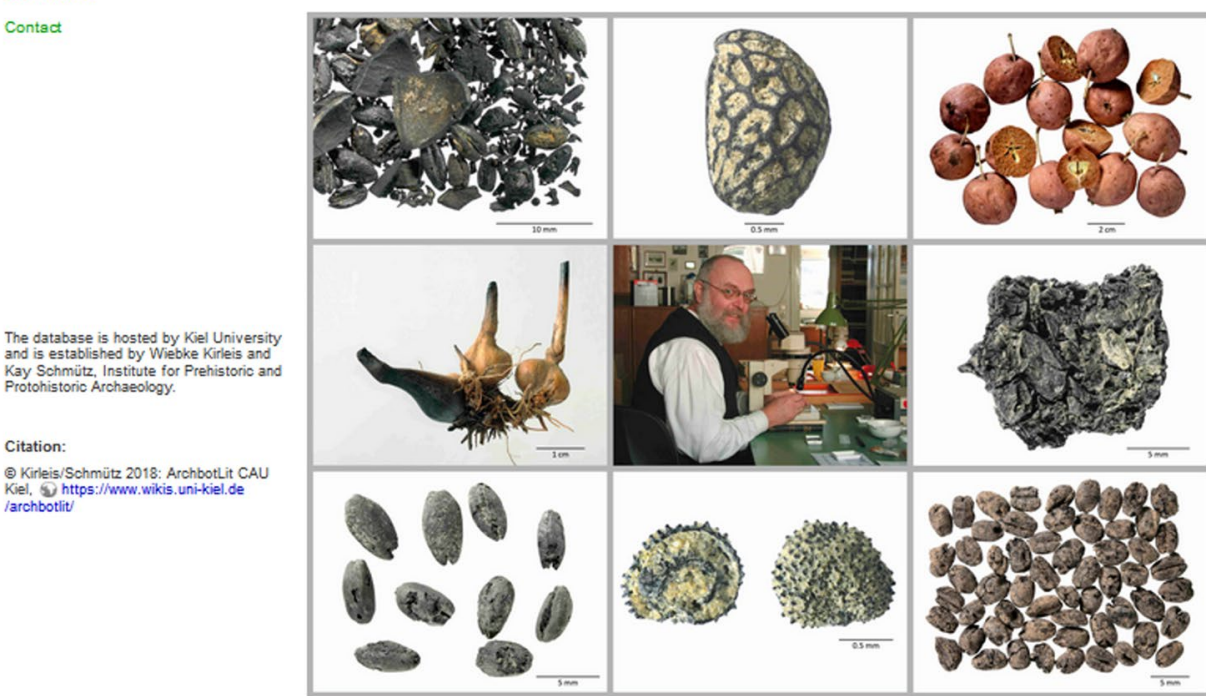

Photos:

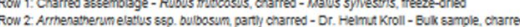

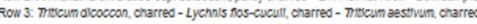

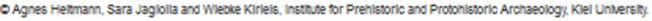

were transferred into the new format, which is accessible at https://www.wikis.uni-kiel.de/archbotlit.

\section{The technical background of the new wiki platform ArchbotLit}

To provide a sustainable solution for data storage and access, we have chosen a wiki platform, DokuWiki (https://www. dokuwiki.org/), as the format for ArchbotLit. A wiki is an unusual choice for a literature database: due to the generally well-structured nature of bibliographic data, it could as well be stored in a relational database system. Nevertheless, we opted for DokuWiki because of the comprehensible underlying file-based back-end, which is a significant advantage for long term and version-independent data storage. It provides a simple (e.g. navigation) and stable front-end, good documentation and is open source software. Contrary to a relational database with a home-made front-end (e.g. Ruby on Rails), it can immediately (i.e. without administrative changes in a database structure) incorporate any kind of additional information beyond the pure bibliographic data.
Users do not need complex training to apply changes to the wiki entries, but can rely on a markup-text based system. DokuWiki further accounts for reasonable narrow digital storage capacities and in the long-run minimizes disturbances by recurrent needs for integration of software updates. A drawback is the minimalistic design of the platform and interfaces, which only offers limited and basic tools to search, view and edit entries.

A major challenge of DokuWiki was its focus on singleentry editing. Usually users are expected to edit each individual entry manually, which is fine for wikis in general, but not sustainable for a literature database where bulk imports are expected to be the main means of data input. In order to solve this problem we developed a mass import software tool written in Python, which we provide with ArchbotLit. We used this software for the initial setup to transform and import data from preceding database implementations. 


\section{The functionality of ArchbotLit}

The ArchbotLit database offers open accessible functions to search in the integrated literature for general and specific parameters upon archaeobotanical data with search options for single as well as multiple variables. The available data consist of secondary information on archaeobotanical evidence of crop species comprising (1) Species, (2) Archaeological period/dating, (3) Country, (4) Geographical coordinates, (5) Site and (6) Reference. Ideally, links are provided to access the respective publications and the raw data on the www.

The most important backbone for the ArchbotLit database is the list of taxa and synonyms that ensures concise data entry and availability. Similar to the parent databases, the predetermined taxalist is based upon the catalogue Mansfeld's World Database of Agricultural and Horticultural Crops, which includes further synonyms (Schultze-Motel 1986) and can be found online at https://mansfeld.ipk-gatersleben.de.
The taxa list has a focus on crop plants and domesticates and it is supplemented by those taxa that are relevant in the context of crop cultivation (e.g. common weed species), or that are considered as potential edible plants (e.g. Nuphar lutea).

With ArchbotLit as the central database it is possible to trace specific taxa, obtain an overview on crop spectra for specific periods or regions and, if the respective information is provided, create compilations on the occurrence of a plant taxon or species according to site, period, culture/ archaeological group, geographic distribution, author/s and year of publication. The search for a taxon first of all provides a hit list for the ArchbotLit taxa and the synonym list as output, followed by a hit list with links to the respective references. Searching for multiple variables directly results in the hit list with the links to the references. For each reference, metadata are provided including a full qualitative list of all the taxa mentioned in the publication (Fig. 2).

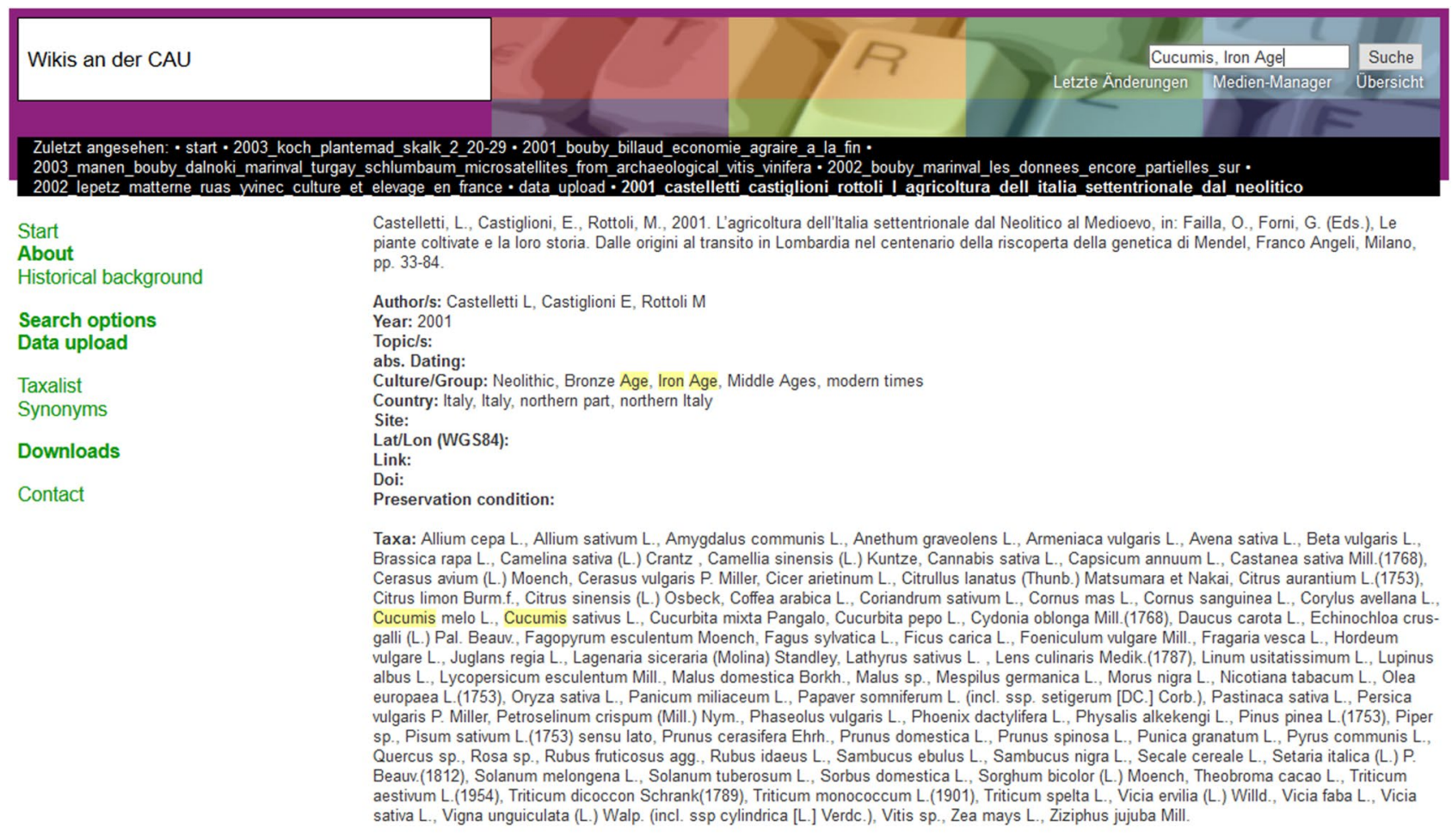

The database is hosted by Kiel University and is established by Wiebke Kirleis and
Kay Schmütz, Institute for Prehistoric and Protohistoric Archaeology.

Citation:

(c) Kirleis/Schmütz 2018: ArchbotLit CAU Kiel,

(6) https://www.wikis.uni-kiel.de/archbotlit/

2001_castelletti_castiglioni_rottoli___agricoltura_dell_italia_settentrionale_dal_neolitico.txt · Zuletzt geändert: 2019/07/01 13:09 von treiser

Fig. 2 Output format for a reference including metadata and a full (qualitative) list of taxa mentioned in the respective publication 
The main change and challenge of the new wiki platform ArchbotLit is that the responsibility for data upload is now handed over to the authors of the respective archaeobotanical publications. This measure was taken in order to cope with the rapidly increasing number of publications in recent years that no longer allow for upload by a single person. Thus, the database relies on the data being uploaded by experts from the archaeobotanical community who have to register once with ArchbotLit. This is done by sending an informal mail to archbotlit@ufg.uni-kiel.de stating "I want to register with ArchbotLit" - a measure to avoid spam entries. Data upload can be carried out with a single record import tool for data from a specific paper, or, to enter multiple records, by use of the Excel-mass-import tool. To facilitate the data upload and ensure the quality of data, taxon names are automatically changed into the taxa list names during the upload procedure, by comparison with the list of synonyms. The synonym list is continuously updated upon request.

\section{Conclusions/outlook}

By now the new database already comprises more than 10,642 species data sets, thanks especially to the extensive engagement of Christoph Brombacher and the team of the unit "Integrative Prehistory and Archeological Science (IPAS)" at Basel University, Switzerland, who have already entered the data of numerous publications. We would like to encourage further authors of new (and old) archaeobotanical publications and archaeobotanical working groups to make use of the updated online platform. This will enable the advertisement of their own publications and increase the relevance of archaeobotanical research on the history of crops by ensuring sustainable data access.
Funding Open Access funding provided by Projekt DEAL.

Open Access This article is licensed under a Creative Commons Attribution 4.0 International License, which permits use, sharing, adaptation, distribution and reproduction in any medium or format, as long as you give appropriate credit to the original author(s) and the source, provide a link to the Creative Commons licence, and indicate if changes were made. The images or other third party material in this article are included in the article's Creative Commons licence, unless indicated otherwise in a credit line to the material. If material is not included in the article's Creative Commons licence and your intended use is not permitted by statutory regulation or exceeds the permitted use, you will need to obtain permission directly from the copyright holder. To view a copy of this licence, visit http://creativecommons.org/licenses/by/4.0/.

\section{References}

Kirleis W, Schmütz K (2018) ArchbotLit CAU Kiel. https://www.wikis .uni-kiel.de/archbotlit/. Accessed 14 Aug 2020

Kroll H (1995) Literature on archaeological remains of cultivated plants (1992/1993). Veget Hist Archaeobot 4:51-66

Schultze-Motel J (1983) Literatur über archäologische Kulturpflanzenreste (1981/1982). Die Kulturpflanze 31:281-297

Schultze-Motel J (ed) (1986) Rudolf Mansfeld, Verzeichnis landwirtschaftlicher und gärtnerischer Kulturpflanzen (ohne Zierpflanzen), 2nd edn. Springer, Berlin, Heidelberg, New York, Tokyo

Schultze-Motel J (1992) Literature on archaeological remains of cultivated plants (1989/1990). Veget Hist Archaeobot 1:53-62

Publisher's Note Springer Nature remains neutral with regard to jurisdictional claims in published maps and institutional affiliations. 